\title{
LA SALUD Y SUS DETERMINANTES SOCIALES Desigualdades y exclusión en la sociedad del siglo XXI
}

\author{
THE SOCIAL DETERMINANTS OF HEALTH \\ Inequalities and exclusion in XXIst century societies
}

\author{
Pedro A. Palomino Moral pamora@ @ujaen.es \\ Universidad de Jaén. España.
}

\author{
María LUISA GRANDE Gascón mlgrande@ujaen.es \\ Universidad de Jaén. España.
}

\author{
Manuel Linares Abad mlinares@ujaen.es \\ Universidad de Jaén. España.
}

\begin{abstract}
RESUMEN
El concepto de salud ha experimentado un proceso de revisión constante. Desde mediados del siglo XX se ha producido un desplazamiento desde la búsqueda de las causas de la enfermedad centradas en el individuo a la aparición de los determinantes sociales, los principales moduladores del fenómeno salud y la enfermedad. Hoy sabemos que la salud y la calidad de vida son un resultado social directamente relacionado con las condiciones generales de la vida de las personas y con la forma de vivir; en este sentido se han hecho notables esfuerzos en las últimas décadas para comprender cómo interactúan los determinantes sociales y se producen los resultados en salud. Analizamos las aportaciones que han conseguido poner de manifiesto los principales factores generadores de las desigualdades sociales incluyendo un análisis de las desigualdades en salud de las mujeres, la vulnerabilidad y el riesgo de exclusión.
\end{abstract}

\section{Palabras Clave}

Desigualdades en salud; Determinantes sociales de la salud; Exclusión social; Promoción de la salud; Salud.

\section{Abstract}

The health concept has undergone a process of constant revision. From the mid-twentieth century it has taken place a shift from the search for the causes of the disease focused on the individual to the emergence of health social determinants that are the main modulators of the health and disease processes. Today we know that health and quality of life are a social result directly related to the conditions of people's life and way of life. In this sense it has been made significant efforts to understand how they interact with the social determinants and how health outcomes occur. We analyzed the contributions that have succeeded in highlighting the main factors of social inequalities including an analysis of inequalities in women's health, vulnerability and risk of exclusion.

\section{KEYWORDS}

Health; Health promotion; Inequality of Health; Social determinants; Social exclusion. 


\section{INTRODUCCIÓN}

La salud es un concepto difícil de definir, a la vez representa un prerrequisito indispensable para satisfacer cualquier tipo de aspiración sobre el bienestar y la felicidad. Ahí reside la importancia de comprender y acotar este concepto, situado en uno de los focos de interés de cualquier sociedad, de cualquier lugar y de cualquier tiempo. Actualmente la salud y la calidad de vida ocupan un lugar central en nuestra sociedad, podemos decir que la sociedad del siglo XXI se encuentra "salutizada" dado que la salud es un derecho, un valor, una aspiración, un recurso, una necesidad y una demanda social que está en el centro de las decisiones en la práctica totalidad de ámbitos públicos y privados. Este trabajo propone una reflexión acerca del proceso de evolución del concepto salud a lo largo del siglo XX hasta la actualidad, proceso caracterizado por el conocimiento científico acerca de los determinantes sociales de la salud, que implica un desplazamiento del foco de atención desde lo personal a lo comunitario y social, donde residen los auténticos determinantes de las desigualdades en salud y la exclusión.

\section{UNA REVISIÓN DEL CONCEPTO DE SALUD, DESDE LO BIOLÓGICO A LO SOCIO-POLÍTICO}

Para responder a cuestiones como cuáles son los factores que influyen sobre la salud y la calidad de vida de las personas y las sociedades, cómo se explica la variabilidad social respecto de la salud o de la esperanza de vida de las personas dentro de un país, entre países o dentro de una ciudad, o cómo superar la brecha de desigualdad entre unos grupos sociales y otros, debemos disponer de marcos de análisis y reflexión que han ido construyéndose sobre el conocimiento sucesivo de las revisiones sobre el concepto de salud y sus determinantes desarrolladas a lo largo del siglo XX hasta la actualidad.

La idea del origen natural de los fenómenos de salud y enfermedad ya estaba presente en el mundo griego; Higia simbolizaba la creencia de que los hombres podían mantenerse sanos si vivían de acuerdo con la razón y el culto de la virtud; el ideal de mens sana in corpore sano de Hipócrates (460 a.c.) consigue explicar la enfermedad a partir de la influencia de factores naturales (Dubos 1987). Sin embargo este conocimiento permaneció olvidado durante gran parte de la Edad Media.

Durante la Revolución Industrial se empieza a abandonar la explicación mágica de las enfermedades, comenzándose a evidenciar asociaciones entre la situación de salud de la población y determinadas condiciones de vida. Los cambios socio-demográficos que acompañaron a la Revolución Industrial trajeron graves consecuencias para la salud de la población: cólera, tifus, tuberculosis, desnutrición infantil, alcoholismo, abandono de niños, y todo ello como resultado del éxodo del campo a la ciudad y los graves problemas de hacinamiento, empobrecimiento y marginación a las que llevaban las malas condiciones de trabajo y de vida. 
Hasta mediados del siglo XX la idea de salud se había caracterizado por su concepción puramente física-biologicista y por ser definida a partir de la enfermedad; es decir la salud era la ausencia de la enfermedad y la atención sobre el binomio salud-enfermedad estaba centrada en explicar las causas de la enfermedad. Para ello se formularon sucesivas teorías, desde la influencia demoníaca o divina, a la humoral, la participación de los miasmas y a la microbiana que finalmente solucionó de una forma definitiva el origen de las principales causas de muerte hasta ese momento: las enfermedades infecciosas (Piédrola Gil 2001).

Ya en el siglo XX las visiones sobre la salud han ido redefiniéndose a la luz de la razón, la reflexión y el debate científico. La Tabla 1 representa las principales concepciones respecto del concepto de salud a lo largo del siglo XX.

Tras la Segunda Guerra Mundial, un conjunto de factores sociales como el avance científico-tecnológico: el conocimiento de la historia natural de las enfermedades y de la forma de prevenirlas, el cambio en el patrón epidemiológico, la influencia de los medios de comunicación, la difusión del conocimiento científico y el fenómeno de la internacionalización de los fenómenos de salud y enfermedad, pusieron de manifiesto la relación entre salud y los determinantes que la influyen. Todas estas causas provocaron que el concepto de salud evolucionara hacia un enfoque positivo, dinámico, multicausal y social. En 1946 la Organización Mundial de la Salud (OMS) define la salud como "El estado de completo bienestar físico, mental y social y no solamente la ausencia de afecciones o enfermedades"; los aspectos innovadores de esta definición hicieron que marcara un hito en su momento con repercusiones importantes que han llegado hasta hoy, en especial el enfoque positivo de la salud. Nos parece importante resaltar que la salud es, por primera vez un derecho humano universal, estando presente en el artículo 25 de

Tabla 1.

Evolución del concepto salud

Concepciones en torno a la salud durante el siglo XX

I. Concepción tradicional. La salud es la ausencia de enfermedades e invalideces, es la vida en el silencio del cuerpo.

II. Salud biestestar. El estado de completo bienestar físico, mental y social y no solamente la ausencia de enfermedades.

III. Salud adaptación, equilibrio, capacidad de funcionar. La salud es el estado de adaptación al medio y la capacidad de funcionar en las mejores condiciones en dicho medio.

IV. Salud como forma de vida. La salud es una forma de vivir que es satisfactoria, autónoma, solidaria y feliz.

V. Salud positiva, potencial personal y factor de desarrollo humano. La salud es la capacidad de desarrollar el propio potencial personal y responder de forma positiva a los problemas del ambiente. 
la Declaración Universal de los Derechos Humanos. Para la OMS el disfrute del más alto nivel posible de salud es un derecho humano fundamental, y debe estar al alcance de todos "sin distinción de raza, religión, ideología política o condición económica o social" (Martínez de Pisón 2006).

Solucionar el problema de las enfermedades ha sido la gran preocupación de la humanidad, lo que ha marcado un sesgo en el estudio y reflexión acerca de los fenómenos salud-enfermedad. Desde hace algunas décadas la salud tiene entidad en sí misma, no sólo se trata de evitar la enfermedad y el sufrimiento sino favorecer la salud y la calidad de vida. La salud como explica Caba (1990), "No es lo opuesto a la enfermedad. El concepto de salud es inseparable de la paz y de la amenaza de guerra, de la eliminación de la pobreza y de la marginación, de la conservación del medio ambiente, de la reducción del desempleo. La salud y la enfermedad no deben concebirse como conceptos estrictamente biológicos e individuales y se deben formular como procesos condicionados por determinadas circunstancias sociales y económicas". En los años siguientes a la formulación clásica de la OMS mencionada antes no faltaron críticas y propuestas revisionistas a la misma, entre ellas la de Milton Terris, quien dice que la salud tiene dos aspectos: "uno subjetivo que se refiere a - sentirse bien - y el otro objetivo que implica -la capacidad para la función —"; también Salleras habla de "el logro del más alto nivel de bienestar físico, mental y social y de capacidad de funcionamiento que permitan los factores sociales en los que viven inmersos el individuo y la colectividad" (Salleras 1986).

De esta manera, las sucesivas reflexiones han ido conectando el proceso saludenfermedad desde lo personal a lo social y comunitario, conexión establecida desde siglos anteriores en el movimiento de medicina social liderado por personas como J.P. Frank o Sigerist (Sigerist 1998). Hernán San Martín (1995) es un destacado salubrista que destaca el carácter social y multifactorial de la salud, por ejemplo afirma que "nos ha costado siglos darnos cuenta de nuestro bienestar biológico, social y mental; que la salud y la enfermedad, no son meros accidentes en nuestra vida, ni tampoco premio 0 castigo que nos caen del cielo, sino que corresponden a situaciones ecológicas, económicas y sociales bien precisas que nosotros mismos, a través de nuestra historia, hemos provocado". De esta manera la salud es entendida como un proceso de adaptación; a partir del cual, salud y enfermedad son fenómenos relacionados con acontecimientos biológicos, la trayectoria personal, el entorno físico, social y cultural de las personas, en una relación que envuelve la vida de las personas. Last, en su diccionario de Epidemiología la define como "equilibrio dinámico en el que la capacidad de un individuo o de un grupo para enfrentarse a todas las circunstancias de la vida se encuentran en un nivel óptimo" (Colomer Revuelta 2000).

Por todo ello, la salud puede ser entendida como una forma de vida y una opción personal 0 una oportunidad de vida plena y gozosa según la visión aportada por Katherine Mansfield (Germán 2000). En 1976 el X Congreso de Médicos y Biólogos de Cultura Catalana, describía la salud como "Una manera de vivir autónoma, solidaria y gozosa"; otros como Iván Illich afirman que "salud es, después de todo, una palabra 
cotidiana que se usa para designar la intensidad con que los individuos hacen frente a sus estados internos y sus condiciones ambientales", incluyendo la noción del equilibrio de las fuerzas vitales, de armonía, de autonomía y responsabilidad en el proceso de adaptación al medio ambiente (Ílich 1978). La propia OMS reformula en los años 90 su idea de salud para expresar que la salud es un concepto positivo entendido como un potencial o capacidad de realización personal que incluye aspectos espirituales (OMS 1998).

En esta traslación, desde el foco de interés centrado en la enfermedad y lo biológico a lo social, hay que citar a un hito en la historia de la Salud Pública, el llamado Informe Lalonde, cuyos resultados ponen de manifiesto el enfoque multicausal del proceso salud-enfermedad a través de la interrelación compleja de cuatro grandes determinantes: la biología humana, el medio ambiente, los estilos de vida y el sistema de atención sanitaria. Este trabajo permitió poner de manifiesto tres hechos relevantes, por un lado el peso o grado de contribución potencial a la mortalidad de la sociedad de aquel tiempo de cada factor determinante -los estilos de vida (43\%), la biología humana (27\%), el medio ambiente (19\%) y el sistema sanitario (11\%) - En segundo lugar, la información aportada indicaba que era epidemiológicamente obvio que las principales causas de enfermedad, muerte y discapacidad eran evitables y que las decisiones y hábitos personales generan riesgos comportamentales aprendidos, y por lo tanto, evitables e intervenibles desde el punto de vista preventivo y educativo. En tercer lugar permitió poner de manifiesto el desfase en la asignación de los recursos dedicados a la salud y la importancia relativa de cada determinante; en este punto la asistencia sanitaria es la receptora de la mayoría de los recursos en detrimento de la promoción de la salud (Salleras 1986). A través de la propuesta de Lalonde, hoy sabemos que el estado de salud de una población está determinado por una serie de elementos (determinantes) que actúan de manera interactiva. Estos factores pueden clasificarse en: a) organización general de un país, política, sociedad, servicios, equidad, justicia, paz, familia, protección social, etc.; b) determinantes demográficos, edad media, distribución de los grupos de edad; c) determinantes biológicos, genéticos; d) determinantes medio ambientales, agua y su calidad, aire, entorno químico y tóxico, energético; e) prosperidad, renta, reparto de la riqueza; f) cohesión social, categorías sociales, desigualdades sociales, relaciones familiares y sociales, etc.; g) sistema educativo, acceso, características; h) valores, nivel de formación y de información, acceso; i) sistema de atención sanitaria, cobertura, calidad, alcance de los mismos, efectos sobre la población, etc.

Durante la década de los años 70 y hasta la actualidad, se han ido acumulando información y evidencia en relación a la variabilidad de los fenómenos de salud y enfermedad en la sociedad, investigaciones que han aportado nuevas evidencias acerca de la importancia de los determinantes sociales de la enfermedad. McKeown en 1979, por ejemplo, puso de manifiesto que el descenso de la tasa de mortalidad debida a las principales enfermedades infecciosas en los últimos dos siglos tuvo lugar antes de desarrollarse las medidas terapéuticas derivadas del descubrimiento de la quimioterapia y la vacunación, es decir, este descenso era debido a causas sociales. Por todo ello, uno de los núcleos 
de interés de las últimas décadas en el ámbito de la Salud Pública se ha polarizado alrededor de los determinantes sociales.

La evidencia de los determinantes sociales de la salud impulsaron durante las últimas décadas del siglo XX el movimiento de Promoción de Salud a través de propuestas internacionales impulsadas desde la OMS entre ellas la Conferencia de Ottawa y la redacción de la Carta de Ottawa de Promoción de Salud en 1986 que merece destacarse por ser una auténtica partida de nacimiento del movimiento de Promoción de Salud (Frías 2000).

La Estrategia de Salud para Todos en el año 2000 formulada por la OMS en el año 1979 o el programa Salud 21 (Ministerio de Sanidad y Consumo 1999) se han establecido en torno a los principios de Promoción de Salud anteriormente expuestos siendo sus ejes vertebrales la salud como derecho fundamental de todos los seres humanos, la equidad en materia de salud y la solidaridad de acción entre todos los países, dentro de ellos y entre sus habitantes (Restrepo 2002).

De forma resumida, se han establecido dos modelos a la hora de intervenir en la mejora de la salud colectiva, bien desde un modelo del déficit (centrado en la enfermedad y en los factores de riesgo) o bien desde un modelo promotor de salud que pone atención en el empoderamiento y la intervención en los entornos vitales de las personas, favoreciendo el control de los individuos y grupos sobre las variables que condicionan su salud. La focalización hacia los factores de riesgo y la enfermedad cuenta con una dilatada trayectoria histórica que ha llegado hasta nuestros días (modelo del déficit), y tiene una consecuencia relevante si consideramos que no incide en los aspectos promotores de la salud (Morgan y Ziglio 2007). Tanto es así que casi toda la evidencia científica que se ha generado en Promoción de Salud lo ha sido desde el modelo del déficit (AlvarezDardet 2011). El modelo del déficit conlleva la minimización del empoderamiento de las poblaciones a las que van dirigidos los esfuerzos en salud de tipo profesionales. Así, los recursos y servicios profesionales, tanto sanitarios como sociales, se siguen enfocando hacia el modelo del déficit. Pero este modelo del déficit está lentamente empezando a girar hacia el otro modelo, es decir, pasando del énfasis en los factores de riesgo y la enfermedad a considerar las ventajas de trabajar con los activos en salud en la sociedad actual (Hernán 2009).

El siglo XXI es un escenario social dominado por los cambios continuados y acelerados en la situación socio-política a escala mundial enmarcados en el fenómeno de la globalización. Los cambios medioambientales y la industrialización son considerados como una nueva amenaza que enmarca la denominada sociedad bautizada por Ulrich Beck como Sociedad del Riesgo (Beck, 1998). Los riesgos son visibilizados mediante la identificación de factores que amenazan la salud humana: carga tóxica, residuos radioactivos, ruidos o emisión de gases invernadero a la atmósfera -entre otros muchos factores - que plantean un entorno físico amenazado y directamente relacionado con importantes problemas de morbi-mortalidad (Unión Europea 2003). Junto a ello coexisten bolsas de pobreza y desigualdad en los países, y marcados desequilibrios en el reparto de la riqueza entre países. La salud humana está también amenazada en las 
dimensiones psicológicas y social, debido a los ritmos de vida: estrés, relaciones humanas, desarraigo, exclusión social, etc. El escenario social está presidido por los cambios demográficos, totalmente necesarios para explicar los patrones de morbilidad, mortalidad, y discapacidad con una notable presencia del fenómeno del envejecimiento. Los cambios demográficos explican en parte la creciente carga de enfermedades crónicas y discapacidad además del volumen progresivamente necesario de recursos dirigidos a atención sanitaria y social. Forma parte destacada de este escenario los cambios sustantivos en la familia, la nuclearización de la misma o la aparición de nuevas formas familiares.

Actualmente asistimos a momentos de crisis y tensiones financieras que desmontan el Estado del Bienestar, la precarización del trabajo y relaciones laborales, con preocupantes tasas de paro que afectan especialmente a la población más joven a la vez que el riesgo de exclusión se extiende a todas las etapas de la vida y a todos los grupos sociales. Todo este escenario queda envuelto por los cambios derivados de la revolución científico-tecnológica y la era de la información digital.

\section{LA BÚSQUEDA DE LOS DETERMINANTES SOCIALES DE LA SALUD Y LAS CAUSAS DE LAS DESIGUALDADES}

El concepto de desigualdad en salud alude al impacto que tienen, sobre la distribución de la salud y la enfermedad en la población, los determinantes sociales como la riqueza, la educación, la ocupación, el grupo racial o étnico, la residencia urbana o rural y las condiciones de habitabilidad o equipamientos del lugar en el que se vive 0 trabaja. La OMS define el concepto desigualdad como las diferencias en materia de salud que son innecesarias, evitables e injustas; por lo tanto es un concepto que incluye una dimensión moral y ética. El fenómeno de las desigualdades en salud está caracterizado según Daponte por su carácter injusto y evitable, además de su ubicuidad, consistencia, su enorme magnitud, su carácter gradual, su tendencia creciente, su persistencia, su carácter histórico y su carácter adaptativo (lo que indica que los grupos sociales mejor posicionados socialmente son los más beneficiados de las acciones sanitarias y sociales dirigidas a mejorar la salud) (Daponte 2009). El concepto es el de equidad en salud y hace referencia a la disminución o ausencia de estas diferencias. Amartya Sen, Premio Nobel de Economía y miembro de la Comisión sobre Determinantes Sociales de la Salud creada por la OMS en 2006 afirma sobre la equidad que no concierne únicamente a salud, vista aisladamente, sino que debe abordarse desde el ámbito más amplio de la imparcialidad y la justicia de los acuerdos sociales, incluida la distribución económica; prestando la debida atención al papel de la salud en la vida y la libertad humanas. La equidad en salud va más allá, por todo ello, de la distribución de la salud y de la asistencia sanitaria o de los recursos (Sen 2002). El "Black Report" (Department of Health 1980) británico es considerado el primer informe sobre la desigualdad del siglo XX, y ya 
en 1980 puso clara la evidencia sobre el riesgo diferencial de muerte según clase social, además del estado de desigualdad de Inglaterra respecto a otros países del entorno. El primer informe similar español data de 1996, el denominado Informe de las Desigualdades Sociales en España (Navarro et al.1996).

Es importante reconocer la dificultad de la tarea de comprender la compleja maraña de causas y efectos en los determinantes de la salud, ya que interactúan entre sí de manera compleja y los resultados en términos de salud son una acción combinada de los mismos. Por ello, explicar las relaciones entre los determinantes es un objetivo fundamental para el apoyo de estrategias de intervención adecuadas y basadas en el conocimiento disponible. En este sentido se han desarrollado varias teorizaciones y propuestas de modelos explicativos acerca de la relación entre desigualdades sociales y salud (Daponte 2009): teoría de los factores psicosociales, selección natural o social, el materialismo y la perspectiva del ciclo vital, etc. Dahlgren y Whitehead (2006) proponen un modelo sobre los determinantes sociales de la salud ampliamente utilizado por la OMS denominado Modelo Socioeconómico de Salud representado en la Figura 1.

Figura 1.

Modelo socioeconómico de Salud de Dalgren y Whitehead. Tomado de Whitehead M, Dahlgren G. 2006. Concepts and principles for tackling social inequities in health, Levelling up (I). Copenhagen: World Health Organization.

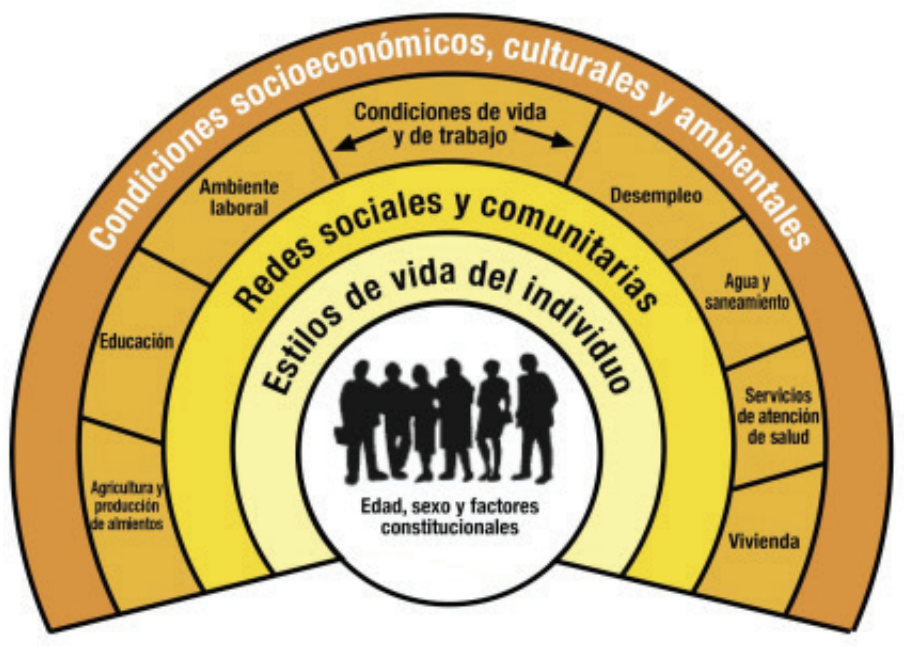


Este modelo trata de representar la interacción de los determinantes sociales. En el centro se encuentra el individuo y los factores constitucionales que afectan a su salud de carácter no modificable. A su alrededor se encuentran en forma de capa los determinantes posibles de modificar, comenzando por los estilos de vida individuales, que son influenciados por la comunidad y las redes sociales. Los determinantes importantes tienen que ver con las condiciones de vida y trabajo, alimentos y acceso a servicios básicos, además de las condiciones socioeconómicas, culturales y ambientales, representadas en la capa más externa.

En la obra de Evans, Frank y Hertzman, que tiene el sugestivo título de ¿Por qué una gente está sana y otra no? (Evans, Barrer y Marmot 1996), se analiza el papel de los grupos sociales y de la segmentación social sobre el desarrollo de los procesos de salud y enfermedad. El grupo y la categorización social (estatus social, ingresos económicos, nivel educativo, ocupación, lugar de residencia) correlacionan con los indicadores clásicos de salud (esperanza de vida, capacidad funcional, accidentes, morbilidad y mortalidad), fenómeno que se conoce como gradiente social. Heterogeneidad social es el concepto utilizado por Evans (1996) para referirse a las diferencias en salud entre los grupos sociales cuyas causas pueden estar asociadas a las características de los propios grupos; tales diferencias suelen recibir el nombre de desigualdades en salud haciendo referencia a su carácter evitable. Una vez establecida la desigualdad y su relación con la heterogeneidad social queda pendiente aclarar el problema de los mecanismos causales. En este sentido Evans et al. (1996) representan el modelo de la heterogeneidad en forma de cubo tridimensional donde el eje $x$ representa la etapa del ciclo vital (perinatal, accidental, enfermedad crónica y senectud); el eje y representa variables grupales (estatus socioeconómico, etnicidad, geográficas, género y grupos con problemas específicos) y el eje $z$ representa las denominadas fuentes de heterogeneidad (causalidad inversa, la susceptibilidad diferencial, la relación entre estilos de vida y salud, el entorno físico, la exposición diferencial, el entorno social y el acceso y la respuesta diferencial de los servicios de salud). En la línea de Evans y colaboradores, Acheson (Gordon 1998) afirma que las desigualdades en salud son la expresión de exposición y vulnerabilidad diferencial que surgen a partir de diferencias en las posiciones socioeconómicas de los individuos, desde la etapa gestacional y durante el curso de la vida, generando interacciones socio - psico- biológicas que además son acumuladas en el tiempo.

Otras propuestas posteriores (Diderischen y Haquist 1998) han intentado establecer los mecanismos por los cuales se establece la compleja relación entre variables psicosociales. La Organización Mundial de la Salud creó la Comisión de Determinantes en Salud con el objetivo de establecer las líneas de actuación contra las desigualdades en salud. De esta manera se desarrolló un marco para el desarrollo de propuestas de intervención contra la desigualdad (Sen 2007) a partir de dos premisas interrelacionada, la primera es que, si efectivamente los principales determinantes de la salud son sociales, aquí deben de estar las soluciones, en el medio social. La segunda premisa es el carácter práctico y aplicado del conocimiento disponible, que tiene que servir para convencer a responsables políticos y sociales de la importancia y relaciones entre salud y políticas públicas. 
El modelo de Solar e Irvin (2007) estructura los factores intervinientes en el proceso de la desigualdad en tres categorías: 1) El contexto socioeconómico y político: la estructura social, la distribución de poder y recursos (gobierno, políticas sociales, derechos, mercado laboral, valores sociales) 2) La estructura social que incluye los distintos ejes de desigualdad que determinan las jerarquías de poder en la sociedad, la clase social, el sexo, la edad, la etnia y el territorio. 3) Los determinantes intermedios: la estructura social determina las desigualdades en los recursos materiales que a su vez influyen en procesos psicosociales y conductas, directamente relacionadas con consecuencias en salud. De esta manera, el modelo explica que la estratificación de los grupos sociales (ingresos, educación, ocupación, género y otros factores) conlleva la creación de inequidades sociales en condiciones de vida y de trabajo. Los mecanismos de estratificación socioeconómica son los denominados determinantes estructurales de inequidades en salud. Son estos mecanismos los que configuran mejores o peores oportunidades para la salud, según diferencias en vulnerabilidades, exposiciones a agentes y acceso a servicios básicos. Es decir, la influencia de la posición socioeconómica del individuo sobre su salud no es directa, sino producto del actuar de factores intermediarios: condiciones materiales, tales como la calidad de vivienda, las circunstancias psicosociales, incluyendo el estrés y los comportamientos como el hábito de fumar o de alimentarse mal. Este modelo incorpora al sistema de salud como un determinante social más; porque, por una parte, el deterioro o mejora del estado de salud tienen un efecto de retroalimentación respecto a la posición socioeconómica, y por otra, el sector salud tiene un rol importante en promover y coordinar políticas de acción sobre determinantes sociales. A estos modelos hay que añadir otros modelos explicativos de las desigualdades en salud, algunos centrados en factores como el género.

\section{Desigualdades de género en SALUd. LA SALUd dE LAS MUJeRES}

En los últimos años se han producido cambios importantes en el paradigma predominante en las Ciencias de la Salud que han tenido una influencia decisiva en la incorporación del enfoque de género en el ámbito de la salud que pone de manifiesto la brecha y desigualdad existente entre hombres y mujeres. En este sentido hay que destacar que los determinantes más importantes que afectan a la salud de las mujeres son precisamente determinantes psicosociales y socioeconómicos. Las desigualdades de género están presentes en todas las sociedades; no hay ningún país en el que las mujeres no sufran algún tipo de discriminación, si bien varía en la forma de manifestarse dichas desigualdades. Tradicionalmente a las mujeres se les ha considerado el "sexo débil" aludiendo con este apelativo a una mayor vulnerabilidad biológica que posteriormente ha sido rebatida con datos estadísticos. En este sentido hay que destacar cómo, por ejemplo, las mujeres tienen una mayor esperanza de vida y, a pesar de que nacen más niños que niñas, éstas sobreviven más que los niños. Esto se traduce en que en la mayor parte de las zonas geográficas del mundo hay 106 mujeres por cada 100 hom- 
bres. Sin embargo se da la paradoja de que aunque las mujeres viven más, su estado de salud es peor y presentan una mayor morbilidad que los hombres (Sánchez 2003); en general las mujeres presentan una prevalencia más alta de enfermedades crónicas y tienen una percepción más negativa de su nivel de salud (Borrel 2004).

A pesar de los importantes avances conseguidos, las mujeres no disfrutan de una igualdad real que les permita tener las mismas oportunidades que los hombres en todos los ámbitos de la vida. Tal y como señala el Programa de Naciones Unidas para el Desarrollo (PNUD) el nivel de salud es un exponente de las desigualdad entre hombres y mujeres de la misma manera que lo es del nivel de desarrollo de un país. Así mismo señala que las desigualdades de género es uno de los obstáculos más importantes para que las mujeres puedan disfrutar en las mismas condiciones que los hombres del derecho a la salud (PNUD 1998). Son los determinantes sociales los que explican las diferencias en la forma de enfermar y morir que existen entre hombres y mujeres, diferencias que a su vez se han utilizado como excusa para justificar la discriminación de las mujeres.

Este enfoque entronca con muchos de los trabajos realizados por Navarro et al. (2007) que defiende que el nivel de salud de la población mejorará en la misma medida en que lo hagan las desigualdades sociales. El análisis de género en salud permite identificar de qué manera los roles sociales, que son atribuidos a hombres y mujeres, y el papel que éstas desempeñan en la sociedad influyen en los procesos de salud y enfermedad de las mujeres. Roles construidos culturalmente, y atribuidos de manera diferenciada a ambos sexos, que sustentan las relaciones de poder en los sistemas patriarcales. Las diferencias y desigualdades sociales se ponen de manifiesto en las diferentes actividades que realizan hombres y mujeres, que son valoradas de manera desigual y que tienen su expresión en el desigual acceso y control de los recursos; en la capacidad para tomar decisiones que les afectan a ellas mismas, a la familia y a la comunidad (San Miguel 2008).

La teoría feminista ha aportado además un marco de análisis que ha permitido visibilizar los determinantes que afectan a la salud de las mujeres y que habían sido ignorados por las teorías predominantes. Como señala Velasco desde la epistemología feminista, "se contempla la salud-enfermedad como un proceso biológico, pero también como expresión de los conflictos por la posición social debida a ser mujer u hombre, los roles de cada sexo y la identidad sexual" y han elaborado "métodos que posibilitan romper las relaciones de subordinación y restablecer la actividad, autonomía (empoderamiento) y socialización" (Velasco 2009).

Las diferentes teorías feministas han realizado contribuciones a los diferentes conceptos de salud que han ido sucediéndose en el tiempo. Una de las principales ha sido la aparición en los años 60 de los Estudios de las Mujeres en la universidad como líneas de estudios e investigación. Posteriormente en los años 80 el género como categoría de análisis es utilizado para poner de manifiesto la influencia de los factores sociales en la salud de los hombres y mujeres. No son sólo las diferencias biológicas lo que explica las desigualdades en la salud de ambos sexos sino que son los factores sociales, culturales, 
políticos, económicos y laborales los que tienen una influencia decisiva en la diferente forma de percibir la salud, de enfermar y de morir de hombres y mujeres (Velasco 2009). Las principales aportaciones feministas en torno a los conceptos de salud y enfermedad han influido en los diferentes modelos de atención sanitaria que han tenido vigencia en diferentes momentos históricos. En este sentido, Velasco señala tres principales líneas que abordan la incorporación del enfoque de género en la salud: salud de las mujeres, desigualdades de género en salud y análisis de género como determinante de salud. Cada una de estas líneas está relacionada con determinadas teorías de salud y con diferentes corrientes feministas. El concepto de salud del que parten influye en los objetivos, los principales temas abordados y en la propuesta que realizan del modelo de atención a la salud de las mujeres.

La primera línea de salud de las mujeres, que se inició en los años 70, hace hincapié en la superación de las desventajas biológicas que suponen algunas funciones reproductivas de las mujeres. Temas como la anticoncepción, el aborto, la maternidad, el embarazo, el parto son recurrentes en este movimiento. La consecución de la liberación y emancipación de las mujeres y la liberación sexual son algunos de los objetivos más conocidos y emblemáticos reivindicados por los movimientos feministas de mediados del siglo pasado. La línea de desigualdades de género en salud se centra en la influencia del contexto social en la salud de las mujeres comparando los diferentes problemas de salud de hombres y mujeres. Se empieza a incorporar el concepto de género en contraposición al sexo, siendo uno de sus principales resultados el inicio de la desagregación de los datos por sexo. Se parte de la premisa de que son las desigualdades sociales y económicas las que explicarían las diferencias en la morbimortalidad diferencial que presentan hombres y mujeres. En este sentido sitúan los factores socioeconómicos en el eje principal, y los múltiples roles que desempeñan las mujeres, en el centro del debate. Otro aspecto importante que se aborda son los sesgos de género en la atención sanitaria, desde el planteamiento de que la salud de las mujeres y los hombres son similares —cuando no es así-, o desde el establecimiento de diferencias - que son inexistentes- y que se acompañan de un trato discriminatorio y un diferente esfuerzo terapéutico en la atención que reciben las mujeres en los servicios sanitarios, discriminación que se extiende también a la investigación en salud (Ruíz 2001) (Ruíz et al. 2004). Este sesgo tiene un exponente grave en la medicalización de algunos de los procesos fisiológicos de las mujeres, como por ejemplo el parto y la menopausia. El análisis de determinantes de género en salud incorpora como eje central el género como categoría de análisis en el proceso de salud y enfermedad de hombres y mujeres, destacando la influencia de los factores psicosociales de género en este proceso. Como indica Velasco (2009:135) "no hay género femenino o masculino, sino modelos de género: tradicional, de transición, contemporáneo, igualitario y otros posibles (...)". Cada uno de estos modelos se acompaña de una serie de representaciones e ideales sociales sobre los roles de hombres y mujeres en la sociedad que configuran la identidad masculina y femenina y que son interiorizados por hombres y mujeres. 
Además de estos determinantes psicosociales hay que destacar la incidencia que los determinantes socioeconómicos y productivos tienen en la salud de las mujeres. El Informe Salud y Género (Ministerio de Sanidad y Consumo 2006), dedicado a las edades centrales de la vida de las mujeres, recoge cuál es la situación en este sentido. Los factores explicativos del impacto diferencial de la salud de las mujeres están relacionados con la doble y a veces la triple jornada de trabajo que soportan las mujeres como consecuencia de su incorporación al mercado de trabajo, la asunción del rol reproductor - que sigue desempeñando casi en exclusividad-, y en el que se incluyen los cuidados familiares (García Calvente 2004). Las medidas de conciliación y corresponsabilidad de la vida laboral y familiar - de las que sólo han hecho uso casi en exclusividad las mujeres-, no han tenido una gran repercusión y en ocasiones han sido negativas para la salud de las mismas, además de que han afectado de manera negativa a su vida laboral (Artázcoz et al. 2001, 2004).

Por otra parte, los fenómenos relacionados con la exclusión social, que tienen un impacto directo sobre los niveles de salud de la población, afectan en mayor medida a las mujeres que a los hombres, tanto en los países ricos como en los países pobres. Estos mayores niveles de pobreza y exclusión social suponen también una limitación en el acceso a los servicios de salud, dándose la paradoja de que quienes más necesitan los servicios de salud son los que menos acceso tienen a los mismos. Tal y como señala Gómez (Gómez 2002:455), "la equidad de género en el ámbito de la salud debe entenderse, entonces, como la eliminación de aquellas disparidades innecesarias, evitables e injustas entre mujeres y hombres que se asocian con desventajas sistemáticas en el contexto socioeconómico". Entendiendo además que la equidad incluye que hombres y mujeres tengan similares niveles de salud y bienestar; que tengan un acceso equitativo a los recursos sanitarios; $y$ que hombres y mujeres tengan las mismas capacidades para participar en la toma de decisiones y una similar distribución de responsabilidades y de trabajo o, como lo denomina Gómez, "la participación para la producción de la salud".

En definitiva la salud de las mujeres además de un componente biológico está condicionada por determinantes psicosociales, y por determinantes socioeconómicos y productivos, siendo además el género un determinante transversal de otros determinantes como son la edad, clase social y etnia que pueden introducir más factores de desigualdad e inequidad que agravan la situación de las mujeres (Esteban 2001) (Borrell et al. 2004).

\section{Hechos probados y evidencia en materia de desigualdades en salud}

Respecto de la cuestión de la medición del fenómeno desigualdad en salud, la literatura presenta varios enfoques: los gradientes de desventaja de la salud y las brechas de salud son las más comunes. El enfoque de salud pública de brechas o diferencias en salud se centra en las diferencias entre los más desfavorecidos y todos los demás y el enfoque del gradiente de salud se relaciona con las diferencias de salud entre todo el espectro de la población, que reconoce un patrón sistemático de desigualdad en salud. 
El más interesante a nuestro juicio es el método del gradiente ya que establece comparaciones entre todos los grupos sociales y presta importancia a considerar un enfoque amplio sobre la sociedad y su diversidad en categorías socioeconómicas, este enfoque está ampliamente promovido por Marmot y su equipo de investigación del Colegio Universitario de Londres y se denomina gradiente social (Wilkinson y Marmot 2003); desde esta perspectiva las inequidades afectan a la jerarquía social en su conjunto y por lo tanto, si las políticas sólo se refieren a aquellos grupos pertenecientes a la parte inferior de la jerarquía social, las desigualdades en salud seguirán existiendo, lo que también significa que los determinantes sociales siguen ejerciendo su influencia. La investigación puesta de manifiesto por Marmot (2005) pone de manifiesto diferencias notables en indicadores clásicos de salud y concentración del riesgo en los grupos más vulnerables; inmersos en situaciones de carencia material y/o social (desempleados de larga duración, inmigrantes, discapacitados, desarraigados, personas sin hogar, pobres, etc.), El círculo vicioso de carencia, marginación, exclusión se asocia a otros efectos como la peor calidad de la vivienda, entornos peor equipados y deteriorados y peor accesibilidad a los servicios de salud.

El informe de la Comisión sobre los Determinantes de la Salud de la OMS denominado "Los hechos probados" (Wilkinson y Marmot 2003) es, probablemente, el documento más difundido sobre la desigualdad en salud a nivel mundial. En él se sintetiza el conocimiento disponible sobre la importancia de diferentes aspectos fundamentales generadores de desigualdad: la primera infancia, la carga de estrés, la pobreza y exclusión social, las condiciones laborales, el desempleo, la red de apoyo social, las drogas, la alimentación y la política de transporte. Esta es posiblemente la visión disponible más amplia de las causas de las desigualdades y exclusión, donde se pueden apreciar un conjunto de factores complejos, dinámicos e interrelacionados.

La exclusión social es un fenómeno social multidimensional que surge como consecuencia de las transformaciones sociales que experimentan las sociedades postindustriales en el marco de la globalización económica (Tezanos 2001), cuyo origen está en los factores relacionados con las causas de la desigualdad anteriormente comentados, entre otros, el aspecto cultural, el económico, el formativo, el sociosanitario, el de vivienda, el relacional y también el espacial. La exclusión social es definida por la Unión Europea (2004) como el resultado de procesos dinámicos y multidimensionales, inducidos por relaciones de poder desiguales, que interactúan a través de cuatro dimensiones fundamentales (económica, política, social y cultural), y a diferentes niveles, incluyendo el individual, hogar, grupo, comunidad y a nivel mundial. El resultado es un continuo inclusión/exclusión caracterizado por un acceso desigual a los recursos, capacidades y derechos, que conduce, entre otros resultados a las desigualdades en salud antes comentadas. La exclusión social aleja a las personas de las oportunidades de empleo, ingresos, educación y formación, así como de las redes y actividades sociales y comunitarias que son claves para el mantenimiento de la salud y de la calidad de vida. Las personas socialmente excluidas apenas tienen acceso a los organismos de poder y de toma de decisiones, por lo que suelen sentirse sin poder e incapaces de tomar el control 
de las decisiones que afectan a su vida cotidiana. La importancia actual del concepto ha generado intentos de acotarlo; en este sentido Tezanos (2004) precisa que se trata de un fenómeno estructural y multidimensional que se lleva a cabo mediante procesos globales que conducen a que ciertos individuos y grupos se encuentren en situaciones que no permiten que las personas disfruten de sus derechos.

En las últimas décadas la sociedad ha experimentado rapidísimos procesos de cambio y transformación que han incidido también en nuevos y cambiantes procesos de desigualdad y exclusión hasta hacerlo un fenómeno donde realmente nadie puede estar a salvo. De esta manera cada persona puede ser "etiquetada" como poseedora de mayor o menor riesgo de exclusión, siendo este un fenómeno diseminado socialmente a partir de la extensión de la precarización. Lejos de la sociedad de décadas pasadas, predecible y sólida alrededor del matrimonio para toda la vida y el trabajo fijo, (donde el riesgo de exclusión estaba relacionado con la vejez y la viudedad), las dinámicas sociales han extendido el riesgo a otras edades como la infancia, la transición de la vida adulta o la vida laboral mediante el fenómeno de la precarización. La difusión del riesgo a lo largo del ciclo de la vida se encuentra además relacionado con las dinámicas familiares y con la capacidad de conciliar las demandas de la esfera personal, familiar y laboral. En este sentido, la familia ha sufrido procesos de transformación notables (incorporación masiva de la mujer al mundo laboral, devaluación de la autoridad parental, la aparición de nuevas formas familiares, cambios en los roles familiares y un notable incremento de rupturas) también relacionados con este fenómeno.

En el Informe sobre la inclusión social en España (Mari-Kloze 2008) se analiza el fenómeno de la exclusión social sobre la perspectiva del ciclo vital humano. Se destaca que el riesgo de exclusión queda condicionado con nuevos procesos con límites difusos donde la precariedad es un concepto nuclear: condiciones de trabajo, salarios bajos, vida familiar, conciliación de la vida personal y familiar, etc. De esta manera se destaca la concentración del riesgo de pobreza en los dos extremos del ciclo de vida: infancia y vejez. Respecto de los mayores presentan los riesgos más elevados de pobreza moderada, si bien se puede reconocer la acción protectora del Estado sobre este grupo social. La nuclearización y distanciamiento de las familias y la tendencia de las generaciones mayores a mantener su independencia residencial contribuyen al debilitamiento de las redes familiares y generan situaciones emergentes de privación de vínculos afectivos y de apoyo que pueden afectar al bienestar de las personas mayores, especialmente cuando se encuentran inmersas en la dependencia funcional. Respecto de la población infantil aquí se concentra alto riesgo de pobreza alta y severa, un riesgo muy relacionado con la composición del hogar (1 o 2 progenitores) y el acceso de los padres al trabajo. El informe citado advierte de que la vulnerabilidad a la exclusión de la población infantil puede ser especialmente lesiva, por cuanto los riesgos sufridos durante la infancia (fracaso escolar, participación en actividades peligrosas, consumo de sustancias adictivas, embarazos prematuros no deseados, situaciones de maltrato o abuso, etc.) se sitúan en la raíz de dinámicas de exclusión que a menudo se arrastran a lo largo de etapas posteriores del ciclo vital (en forma de carencias formativas básicas, paro, trastornos 
psicológicos y de conducta, etc.). En la relación entre género y exclusión se destaca que ser mujer, mayor, y vivir sola aumenta de forma considerable el riesgo de pobreza. También se destaca el riesgo de fractura social derivado de las condiciones de vida de la población inmigrante respecto a la autóctona, con elevada concentración de pobreza más intensa, mayores dificultades económicas en sus hogares, mayores tasas de paro, peores condiciones laborales y mayor exposición de los hijos a la pobreza. Respecto de los jóvenes, su nivel de bienestar está relacionado con su dependencia familiar y económica del hogar familiar de origen. Se afirma en el informe que si se emanciparan siguiendo el modelo tradicional de una pareja con un solo sustentador, siete de cada diez jóvenes que siguen viviendo con sus padres serían pobres. En la actualidad el bienestar de los jóvenes está fuertemente intervenido por la familia, lo que lleva aparejado frustración, baja nupcialidad, baja fecundidad, etc. Queda patente que el riesgo de exclusión se ha extendido a la población trabajadora, donde la precariedad en las condiciones de trabajo y bajos salarios afecta a jóvenes, mujeres e inmigrantes.

\section{DisCAPACIDAD Y EXCLUSIÓN}

Las personas con discapacidad son identificadas como colectivo vulnerable a la exclusión social identificados por el Ministerio de Trabajo y Asuntos Sociales español, además de personas mayores, infancia y familias en situación o riesgo de exclusión, personas drogodependientes, enfermos de sida, población inmigrante, solicitantes de asilo, población reclusa, colectivo gitano, mujeres en situación o riesgo de exclusión y personas sin hogar (Moriña Díez 2007).

En el Informe Mundial sobre la discapacidad (OMS 2011) se ponen de manifiesto los principales obstáculos para este grupo, como la ausencia o insuficiencia de normativa legal relacionada con el acceso a la educación, los servicios de apoyo y protección social, la existencia de creencias y prejuicios por lo que se impide el acceso al trabajo, la atención de salud o la participación social, la prestación insuficiente de servicios, siendo personas vulnerables a errores y deficiencias provenientes de los servicios de atención a la salud, rehabilitación, asistencia y apoyo. También se identifican problemas con la prestación de servicios, entre ellos la mala coordinación, los problemas de dotación, la ausencia de formación o competencia que afectan a las oportunidades de accesibilidad e idoneidad. Además se indican los problemas derivados de la financiación y la sostenibilidad. La accesibilidad es un factor de primera magnitud según este informe, a los edificios, centros de trabajo, lugares públicos, al transporte y a la información. También se identifica la falta de participación en la toma de decisiones. Finalmente se destacan la ausencia de datos sobre la discapacidad y efectividad de los programas e intervenciones.

El conocimiento acerca de la relación entre discapacidad y exclusión pone de manifiesto de forma consistente que los ingresos medios por familia son sustancialmente 
menores en una familia con una persona con discapacidad, existiendo relación entre gravedad de la discapacidad y el grado de pobreza y exclusión. Las mujeres con discapacidad viven frecuentemente en pobreza y en situaciones de exclusión social. También se ha descrito la relación entre la institucionalización de las personas con discapacidad como factor de exclusión social.

En el estudio del CERMI (2003) acerca de la discapacidad y exclusión social en la Unión Europea, se ofrecen datos sobre la importancia relativa de los principales efectos de la exclusión en las personas con discapacidad. Los resultados indican el siguiente orden de importancia: falta o acceso limitado al entorno social y desempleo (94\%), falta 0 acceso limitado a los servicios $(85,4 \%)$, estigmatización de las personas discapacitadas $(83,3 \%)$, falta de formación adecuada $(81,2 \%)$, falta de servicios especializados $(80 \%)$, sistemas educativos inadecuados $(77,1 \%)$, falta de políticas económicas para compensar el gasto extra de la discapacidad $(75,7 \%)$, la estructura del sistema de subsidios $(75 \%)$, vivir en instituciones (60\%). Además, la situación también tiene un gran impacto sobre los padres (la mayor parte madres) de personas con discapacidad severa, que deben compensar esta falta en detrimento de su trabajo, ingresos y derechos de pensión, pero también de su propia participación en la comunidad y entorno social.

El futuro respecto de la cuestión de la atención al colectivo de personas con discapacidad —asumiendo la heterogeneidad en sus causas y condiciones- es complejo. Siendo realistas hemos de considerar que el desarrollo de la ley 39/2006 de Promoción de las Autonomía personal y Atención a las personas en situación de dependencia (BOE 2006) abrió expectativas en relación a sus efectos sobre el grupo de personas con discapacidad mediante acciones favorecedoras de la autodeterminación de las personas como forma de potenciación de la calidad de vida (Lachapelle 2005), sin embargo asistimos al progresivo desmantelamiento del Estado de Bienestar y al desarrollo de la ley, que considera el acceso a las prestaciones como un derecho subjetivo que además precisa el desarrollo de programas, servicios y prestaciones diferenciados. Considerando el desmantelamiento del Estado de Bienestar, la reducción del gasto social y la inercia privatizadora de los servicios públicos los derechos sociales pueden ser derechos inmateriales debido a un escaso desarrollo de programas y/o la financiación reducida.

\section{Consideraciones finALES}

El estudio de la evolución del concepto de salud desde el concepto centrado en la enfermedad al concepto multidimensional de la salud y su relación con los determinantes sociales ha hecho posible establecer un debate en relación a los factores explicativos de las desigualdades en salud incluyendo la identificación de los factores sociales que explican el origen de las desigualdades sociales o el gradiente social. El enfoque de

género en la investigación sobre salud ha evidenciado la brecha existente entre la salud 
de hombres y mujeres, brecha sólo explicable en términos de diferencias de roles, actividades, distribución de recursos, poder y toma de decisiones.

También hay evidencia del mayor riesgo de exclusión en las mujeres y en las personas discapacitadas. La mujer está afectada en mayor medida que el hombre del riesgo de exclusión debido a su desequilibrada posición en la sociedad actual (producción, distribución y consumo de servicios), en el marco de la globalización económica, del debilitamiento de los derechos sociales y del efecto protector del Estado de Bienestar.

\section{ReferenCIAS BibliogRÁFICAS}

Álvarez-Dardet, C. Ruiz Cantero MT. 2011. "Patrimonio de salud: ¿son posibles las políticas salutogénicas?". Revista Española de Salud Pública 2:123-127.

Artázcoz, L. et al.. 2001. "Trabajo doméstico, género y salud en población ocupada". Gaceta Sanitaria 15:150-153

Artázcoz, L. et al. 2004 "Género, trabajos y salud en España”. Gaceta Sanitaria 18: 24-35.

Beck, Ulrich. 1986. La sociedad del riesgo. Hacia una nueva modernidad. Barcelona: Paidós.

Borrell, C. et al. 2004."Desigualdades en salud según la clase social en las mujeres. ¿Cómo influye el tipo de medida de la clase social?". Gaceta Sanitaria 18:75-82.

Borrell, C., García-Calvente María M., Martí-Boscà J. V. 2004. "La salud pública desde la perspectiva de género y clase social". Gaceta Sanitaria 1:2-6.

Caba, P. 1990. "Salud para todos". Pp. 9-22 en VV. AA. La sanidad española a debate. Madrid: Federación Española Defensa de la Sanidad Publica.

CERMI. 2003. Discapacidad y exclusión social en la Unión Europea. Madrid: Cermi.

Colomer Revuelta, C., Álvarez Dardet, C. 2000. Promoción de Salud y cambio social. Barcelona: Masson.

Daponte Codina, A., Bolívar Muñoz, J., García Calvente, M. M. 2009. Las desigualdades sociales en salud. Granada: Escuela Andaluza de Salud Publica.

Department of Health and Social Security. 1980. Inequalities in Health: Report of a Research Working Group. Londres: DHSS.

Diderichsen, F., Haquist, J.1998. "Social inequalities in health: some methodological considerations for the study of societal position and social context". Pp. 25-39 en Arve-Parès B (ed.) Inequality in Health - Swedish Perspective. Estocolmo: Swedish Council for Social Research.

Dubos, R.J. 1987. Mirage of Health: Utopias, Progress and Biological Change. Nueva York: Rutgers University Press.

European Commision. 2004. Joint Report on Social Inclusion. Bruselas: European Commision.

España. 2006. "Ley 39/2006, de 14 de Diciembre, de Promoción de la Autonomía Personal y Atención a las Personas en situación de dependencia". Boletín Oficial del Estado 299:44142-44156, 15 de diciembre de 2006. 
Esteban Galarza. M. L. 2003. "El género como categoría analítica. Revisiones y aplicaciones a la salud". Cuadernos de Psiquiatría Comunitaria 122-39.

Esteban Galarza, M.L.2001. "El género como categoría analítica. Revisiones y aplicaciones a la salud", Pp. 25-34 en Perspectivas de género en salud. Fundamentos científicos y socioprofesionales de dife-rencias sexuales no previstas. Madrid: Minerva Ediciones.

Evans, R., Barrer, M., Marmor, T. 1996. ¿Por qué una gente está sana y otra no? Madrid: Díaz de Santos.

Frías Osuna, A., Palomino Moral, P.A. 2000. "Concepto de Promoción de Salud". Pp. 49-59 en Frías Osuna (Coord.), Salud pública y educación para la salud. $1^{a}$ Edición. Barcelona: Masson.

García Calvente, M.M. 2004. "El impacto de cuidar en la salud y la calidad de vida de las mujeres", Gaceta Sanitaria 18: 83-92.

Germán Bes, C. Marcén Letosa, J.J. 2000. "Nuevo paradigma de salud". Pp. 15-27 en Salud pública y educación para la salud. $1^{\text {a }}$ Edición. Barcelona: Masson.

Gómez, E. 2002. "Equidad, género y salud: retos para la acción". Revista Panameña de Salud Publica 11(5): 454-461.

Gordon, D. 1999. Inequalities in Health: The Evidence; Presented to the Independent Inquiry Into Inequalities in Health, Chaired by Sir Donald Acheson. Londres: The Policy Press.

Hernán García M, Lineros González C. 2009. "Los activos para la salud. Promoción de la salud en contextos personales, familiares y sociales". Fundesfam 2(2): 1-6.

Illich, I. 1978. Némesis médica. México: Editorial Joaquín Mortiz-Planeta.

Lachapelle, Y., Wehmeyer, M. L., Haelewyck, M-C., Courbois, Y., Keith, K. D., Schalock, R., Verdugo, M. A. y Walsh, P,N. 2005. "The relationship between quality of life and self-determination: an international study". Journal of Intellectual Disability Research 49:740-744.

Marí-Klose, M. 2008. Informe sobre la inclusión social en España. Barcelona: Caixa Catalunya.

Marmot, M. 2005. "Social determinant of health inequalities". Lancet 365:1099-1104

Martínez de Pisón J. 2006. "El derecho a la salud: un derecho social esencial". Derechos y Libertades $14: 129-150$

Ministerio de Sanidad y Consumo. 2006. Informe Salud y Género 2006. Las edades centrales de la vida. Madrid: Ministerio de Sanidad y Consumo.

Morgan, A., Ziglio, E. 2007. "Revitalising the evidence base for public health: an assets model". Promotion \& Education 2:17-22.

Ministerio de Sanidad y Consumo-OMS. 1999. Salud 21: Salud para todos en el siglo XXI. Madrid: Ministerio de Sanidad y Consumo.

Moriña, A. 2007. La exclusión social: análisis y propuestas para su prevención. Madrid: Fundación Alternativas.

Moriña, A. 2007. La exclusión social: análisis y propuestas para su prevención. Madrid: Fundación Alternativas. 
Navarro, V., Benach, J, y Comisión Científica de Estudios de las Desigualdades Sociales en Salud en España. 1996. Las desigualdades sociales en salud en España. Madrid: Ministerio de Sanidad y Consumo.

Navarro, V. et al. 2007. "El impacto de la política en la salud". Salud Colectiva 3: 9-32.

OMS. 1998. Glosario de Promoción de Salud. Ginebra: OMS.

OMS 2011. Informe Mundial sobre la discapacidad. Ginebra: OMS.

Piédrola, Gil et al.. 2001. Medicina preventiva y salud pública. Barcelona: Masson.

Programa de Naciones Unidas para el Desarrollo. 1995. Informe sobre Desarrollo Humano. México: PNUD.

Restrepo, H., Málaga, H. 2002. Promoción de Salud, cómo construir una vida saludable. Bogotá: Editorial Médica Internacional.

Ruíz M.T. 2001. "Igualdad de oportunidades en los servicios sanitarios: sesgos de género como determinante de salud de la comunidad". Pp. 163-178 en Miqueo, C. y et al.. Perspectivas de género en salud. Fundamentos científicos y socioprofesionales de diferencias sexuales no previstas. Madrid: Minerva Ediciones.

Ruíz, M.T., Verdú, M. 2004. "Sesgo de género en el esfuerzo terapéutico". Gaceta Sanitaria 18: 118-25

Salleras Sanmartí. L. 1986. Educación Sanitaria: principios, métodos y aplicaciones. Barcelona: Díaz de Santos.

San Martín H. 1995. Salud y enfermedad, México: La prensa médica mexicana.

San Miguel N. 2008. Estrategia. Género en Desarrollo de la Cooperación Española. Madrid: Ministerio de Asuntos Exteriores y Cooperación.

Sánchez MP. 2003. Mujer y salud. Familia, trabajo y sociedad. Madrid: Díaz de Santos.

Sen A. 2002. “¿Por qué la equidad en salud?”. Revista Panameña de Salud Pública 5-6: 302-309.

Sigerist, H. 1998. Hitos de la historia de la salud pública. México: Siglo XXI.

Solar, O. y Irwin, A. 2007. A Conceptual Framework for Action on the Social Determinants of Health. Ginebra: WHO Commission on Social Determinants of Health.

Tezanos, J. F. 2001. La sociedad dividida: estructuras de clases y desigualdades en las sociedades tecnológicas. Madrid: Biblioteca Nueva

Tezanos, J.F. 2004. Tendencias en desigualdad y exclusión social. Madrid: Editorial Sistema

Unión Europea. 2003. "Estrategia Europea de medio ambiente y salud". Bruselas: Comunicación al Consejo, al Parlamento Europeo y al Comité Económico y Social Europeo.

Velasco, S. 2009. Sexos, género y salud. Teoría y métodos para la práctica clínica y programas de salud. Madrid: Minerva ediciones.

Whitehead, M. 1992. "The concepts and principles of equity and health". International Journal of Health Services 22:429-45.

Whitehead, M. y Dahlgren, G. 2006. Concepts and Principles for Tackling Social Inequities in Health Levelling up (I). Copenhague: World Health Organization. Copenhagen. 
Wilkinson, R. G. y Marmot, M. (Eds.). 2006. Los Determinantes sociales de salud: los hechos probados. Organización Mundial de la Salud: Ministerio de Sanidad y Consumo.

Pedro A. Palomino Moral es enfermero y psicopedagogo. Actualmente es Profesor Titular en el Departamento de Enfermería de la Facultad de Ciencias de la Salud de la Universidad de Jaén.

María LuISA Grande Gascón es enfermera y socióloga, Profesora Titular en el Departamento de Enfermería de la Facultad de Ciencias de la Salud de la Universidad de Jaén.

Manuel Linares Abad es enfermero y antropólogo, Profesor Titular en el Departamento de Enfermería de la Facultad de Ciencias de la Salud de la Universidad de Jaén.

RECIBIDO: 16/02/2013

ACEPTADO: 11/02/2014 